\title{
Advantages of colour flow imaging in the diagnosis of left ventricular pseudoaneurysm
}

\author{
GEORGE R SUTHERLAND, $†$ JOHN H SMYLLIE, $\dagger$ JOS R T C ROELANDT * \\ From the ${ }^{\star}$ Thoraxcenter, Academic Hospital Rotterdam-Dijkzigt and Erasmus University, Rotterdam, \\ The Netherlands, and †Department of Cardiology, Southampton General Hospital, Southampton
}

SUMMARY Eleven cases of left ventricular pseudoaneurysm in nine patients were studied by cross sectional echocardiography, conventional Doppler echocardiography, and colour flow imaging. In two patients recurrent pseudoaneurysms developed after cardiac surgery, three had acute rupture after myocardial infarction, two were the result of stab wounds, one was a late rupture of a true left ventricular aneurysm, one developed after surgical resection of a true left ventricular aneurysm, and two as a consequence of left ventricular venting. In all 11 cases the diagnosis was confirmed by angiographic or surgical information or both. The diagnosis was suspected clinically in only four cases. Cross sectional echocardiography alone confirmed the diagnosis in five cases. Neither pulsed wave Doppler nor continuous wave Doppler established the diagnosis when they were used without colour flow imaging in five and three cases respectively. In all 11 cases colour flow imaging showed flow in and out of the pericardial cavity at the defect site as well as the abnormal flow within the pseudoaneurysm. Subsequent use of pulsed Doppler showed a consistent "to and fro" flow pattern across the myocardial defect with characteristic respiratory variation of the peak systolic velocity. This unique intrapericardial flow pattern is diagnostic of a pseudoaneurysm.

Colour flow imaging is a valuable addition to cross sectional and Doppler echocardiography, and is the best technique for detecting left ventricular pseudoaneurysms.

A pseudoaneurysm of the left ventricle is a rare complication of myocardial rupture. Containment of the resulting haematoma by adherent pericardium prevents immediate tamponade and sudden death. ${ }^{12}$ Myocardial infarction is the most common cause of myocardial rupture, which can also be a consequence of ventriculotomy and valve replacement (along the valve annulus), penetrating chest injury, and endocarditis. ${ }^{3}$ The tendency for pseudoaneurysms of the left ventricle to rupture spontaneously has been suggested by pathological studies, ${ }^{45}$ whereas true left ventricular aneurysms tend not to rupture. Therefore, the non-invasive recognition of these two types of aneurysm may be useful and relevant to outcome.

Cross sectional echocardiography is helpful in

Requests for reprints to Dr George R Sutherland, Thoraxcentre, Academic Hospital Dijkzigt, 3000 DR Rotterdam, The Netherlands.

Accepted for publication 24 August 1988 distinguishing between pseudoaneurysms and true aneurysms of the left ventricle. ${ }^{6-8}$ However, unless cross sectional echocardiography shows a communication between left ventricle and the pseudoaneurysm sac the diagnosis cannot be confirmed. Pulsed Doppler can show abnormal flow across the myocardial defect into the suspected pseudoaneurysm and thus prove the diagnosis. ${ }^{910}$ Colour flow imaging is particularly useful for the diagnosis of abnormal intracardiac shunts because it overlays flow information on the cross sectional image. It should therefore aid the diagnosis of cardiac rupture because it improves the definition of the site of transmyocardial flow.

We studied 11 cases of left ventricular pseudoaneurysm (nine patients) diagnosed by colour flow imaging and we describe the diagnostic features of cross sectional imaging and Doppler echocardiography and the advantages of the colour flow imaging technique over both other ultrasound techniques and left ventricular angiography. 


\section{Patients and methods}

\section{PATIENTS}

During a two year period we studied nine consecutive patients (from two cardiac centres) with left ventricular pseudoaneurysm by a combination of cross sectional echocardiography, conventional pulsed and continuous wave Doppler, and colour flow imaging. In two of these patients a recurrent or new pseudoaneurysm developed after operative repair. Table 1 shows the cause of pseudoaneurysm and clinical information on the group. There were three cases of acute rupture after myocardial infarction, two after stab wounds to the chest, one case of late rupture of a true left ventricular aneurysm, one case developed after ventriculotomy for resection of a true left ventricular aneurysm, and two cases resulted from left ventricular venting at aortic valve replacement. In the two cases of recurrent pseudoaneurysm, one had a persisting pseudoaneurysm at the same site and the other had a further distal myocardial rupture after cardiac surgery. In the three patients whose pseudoaneurysm followed myocardial infarction, the time from acute infarction to presentation ranged from three months to four years. The patient with rupture of a true left ventricular aneurysm presented 10 years after the preceding infarction. One of the two cases caused by left ventricular venting presented a year after operation. By contrast the two patients with penetrating chest injury presented within $\mathbf{4 8}$ hours, and two of the three patients with pseudoaneurysm after cardiac surgery and the two patients with recurrent pseudoaneurysms were all diagnosed within the first postoperative week. The diagnosis was suspected clinically in only four cases and there was a localised precordial systolic/diastolic "to and fro" murmur in three cases. Five cases had clinical and radiographic features of left ventricular failure.

Table 1 Cause of pseudoaneurysm and clinical information on 11 cases

\begin{tabular}{|c|c|c|c|c|}
\hline Cause & No & Presentation & $\begin{array}{l}\text { Left } \\
\text { ventricular } \\
\text { failure }\end{array}$ & $\begin{array}{l}\text { Systolic/ } \\
\text { diastolic } \\
\text { murmur }\end{array}$ \\
\hline Post infarct & 3 & $\begin{array}{l}\text { Late ( } 3 \\
\text { mnths }-4 \text { yr) }\end{array}$ & 2 & 2 \\
\hline $\begin{array}{l}\text { Chest injury } \\
\text { Ruptured LV } \\
\text { aneurysm }\end{array}$ & $\begin{array}{l}2 \\
1\end{array}$ & $\begin{array}{l}\text { Early }(<48 \mathrm{~h}) \\
\text { Late }(10 \mathrm{yr})\end{array}$ & $\begin{array}{l}1 \\
1\end{array}$ & $\begin{array}{l}1 \\
0\end{array}$ \\
\hline $\begin{array}{l}\text { Post cardiac } \\
\text { surgery }\end{array}$ & $3(2)$ & $\begin{array}{c}\text { Early (1-8 } \\
\text { days) }\end{array}$ & 1 & 0 \\
\hline $\begin{array}{l}\text { Recurrent } \\
\text { pseudoaneurysm }\end{array}$ & $2^{(1)}$ & $\begin{array}{l}\text { Late (1 yr) } \\
\text { Early (3-8 } \\
\text { days) }\end{array}$ & $\begin{array}{l}\mathbf{0} \\
\mathbf{0}\end{array}$ & $\begin{array}{l}\mathbf{0} \\
\mathbf{0}\end{array}$ \\
\hline Total & 11 & & 5 & 3 \\
\hline
\end{tabular}

LV, left ventricular.

\section{METHODS}

Echocardiographic studies were performed by either a Toshiba SSH $65 \mathrm{~A}$ or a Vingmed 700 colour flow imaging system. Cross sectional echocardiography was performed in the standard parasternal, apical, and subcostal views. When an extracardiac but intrapericardial echo free space that suggested a left ventricular pseudoaneurysm was visualised, an attempt was made to identify any site of myocardial rupture. If a communication between the left ventricle and the echo free space was detected, pulsed and continuous wave Doppler and colour flow imaging studies were concentrated over this area. If no communication was visualised the area of myocardium adjoining the left ventricle and the echo free space were searched for transmyocardial flowinitially by pulsed Doppler and then by continuous wave Doppler and finally by colour flow imaging. Colour flow imaging was performed in the velocity or turbulence mode with appropriate gain and filter settings. If colour flow imaging was successful but conventional Doppler was initially unsuccessful in locating the flow disturbance associated with myocardial rupture conventional Doppler was repeated after realignment through the area of disturbed colour flow to record the Doppler flow pattern.

\section{Results}

CROSS SECTIONAL ECHOCARDIOGRAPHY (Table 2) In 10 of the 11 cases we found a significant extramyocardial echo free space within the pericardial cavity. The site of this space was posterolateral in seven cases and apical in three cases. This extracardiac echo free space was unequivocally continuous with the left ventricular cavity in only five cases. In a further two cases the site of myocardial rupture was equivocal on imaging and in three cases no

Table 2 Echocardiographic data on 11 cases of pseudoaneurysm

\begin{tabular}{|c|c|c|c|c|c|}
\hline Cause & No & $\begin{array}{l}\text { Unequi- } \\
\text { vocal } \\
C S E\end{array}$ & ${ }_{C W}^{\text {Diagnostic }}$ & $\begin{array}{l}\text { Diagnostic } \\
P W\end{array}$ & $\begin{array}{l}\text { Diagnostic } \\
\text { CFI }\end{array}$ \\
\hline $\begin{array}{l}\text { Post infarct } \\
\text { Chest injury }\end{array}$ & $\begin{array}{l}3 \\
2\end{array}$ & $\begin{array}{l}1 \\
1\end{array}$ & $\begin{array}{l}2 \\
1\end{array}$ & $\begin{array}{l}2 \\
0\end{array}$ & $\begin{array}{l}3 \\
2\end{array}$ \\
\hline $\begin{array}{l}\text { Rupture LV } \\
\text { aneurysm }\end{array}$ & 1 & 1 & 1 & 1 & 1 \\
\hline $\begin{array}{l}\text { Post cardiac } \\
\text { surgery }\end{array}$ & 3 & 2 & 2 & 2 & 3 \\
\hline $\begin{array}{l}\text { Recurrent } \\
\text { pseudoaneurysm }\end{array}$ & 2 & 0 & 2 & 1 & 2 \\
\hline Total & 11 & 5 & 8 & 6 & 11 \\
\hline
\end{tabular}

CSE, cross sectional echocardiography; CW, continuous wave Doppler; PW, pulsed wave Doppler; CFI, colour flow imaging; LV, left ventricular. 
communication with the extra-myocardial echo free space could be visualised. In the remaining case the pseudoaneurysm cavity was not imaged at the initial study because it was in a very posterolateral position within the pericardial cavity. The pseudoaneurysm was noted only on subsequent colour flow studies when disturbed flow was seen within the posterolateral myocardium, and when the transducer angulation was changed the flow was seen to enter a posterolateral pseudoaneurysm.

Because most pseudoaneurysms occurred in a posterolateral position, subcostal views were obtained in all cases-because in theory this view might increase the diagnostic accuracy of the technique. However, it was not of any additional diagnostic benefit. The presence of thrombus within the pseudoaneurysm cavity was noted in only six cases, occurring in four of the five pseudoaneurysms that presented late and in only two of the six pseudoaneurysms that presented early.
PULSED AND CONTINUOUS WAVE DOPPLER

By identifying the diagnostic transmyocardial flow pattern, both pulsed and continuous wave Doppler confirmed the diagnosis in the five cases with unequivocal cross sectional images. In the remaining six cases continuous wave Doppler made the diagnosis in three whereas pulsed Doppler made the diagnosis in only one of these three cases. In the three $(27 \%)$ remaining cases cross sectional imaging and conventional Doppler did not establish the diagnosis. After colour flow imaging had localised the myocardial defect, realignment of continuous wave Doppler confirmed the diagnostic flow pattern in all 11 cases whereas pulsed Doppler still failed to show clear diagnostic waveforms in two cases.

The characteristic waveform obtained by both pulsed and continuous wave Doppler consistently showed a multiphasic "to and fro" flow pattern (fig 1). In this example flow into the pseudoaneurysm had two major peaks-the first peak corresponded with

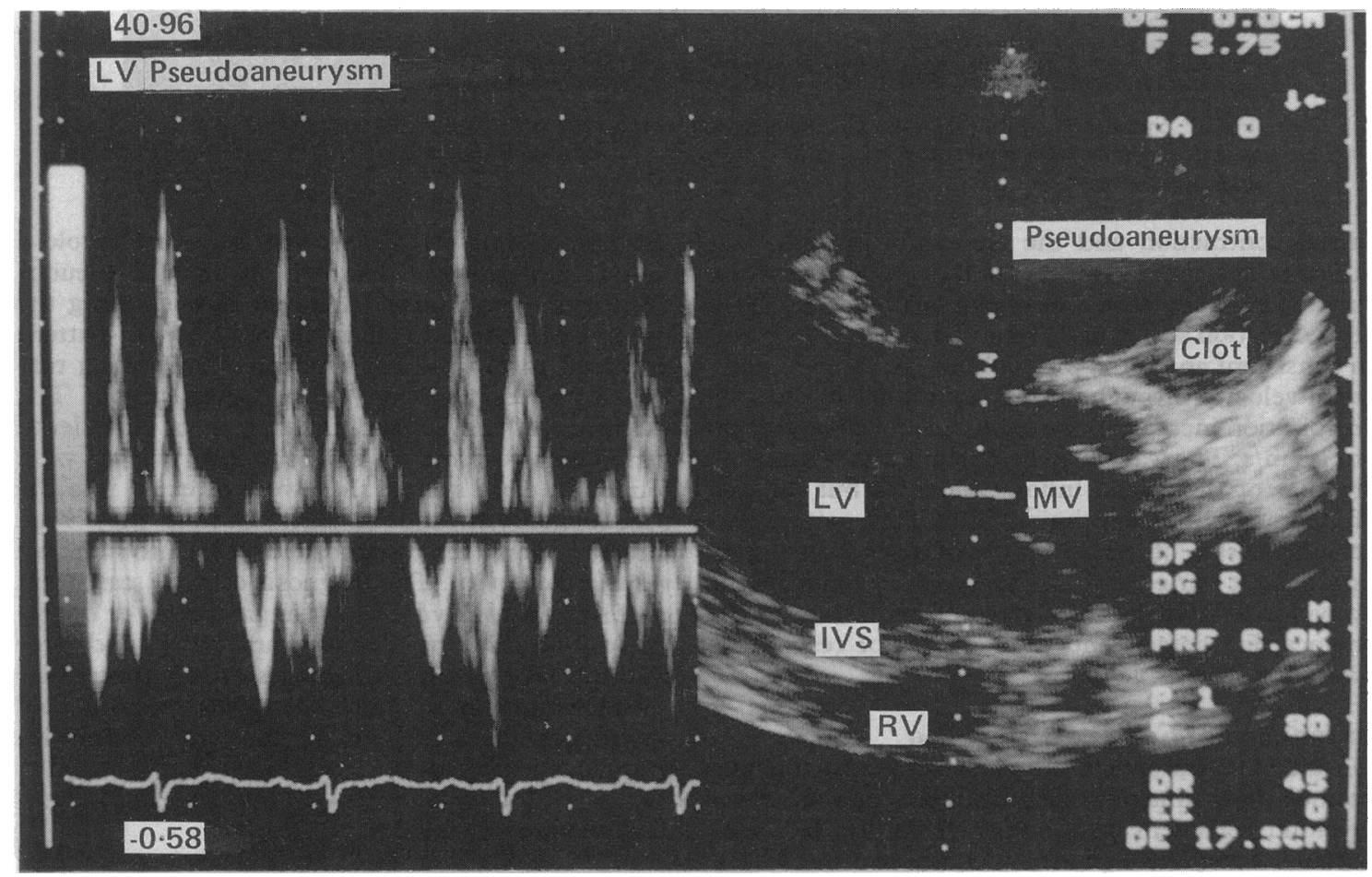

Fig 1 Pulsed Doppler recording from a patient with a left ventricular pseudoaneurysm. The cross sectional image was recorded in a modified four chamber view from a mid-axillary position. The cardiac chambers are displaced by a large posterolateral pseudoaneurysm. The pulsed Doppler sample volume has been positioned at the break in the myocardium. The resulting pulsed Doppler waveform is recorded on the left. Velocities above the zero line indicate flow into the pseudoaneurysm. See text for description of the waveform. $L V$, left ventricle; $M V$, mitral valve; IVS, interventricular septum; $R V$, right ventricle. 


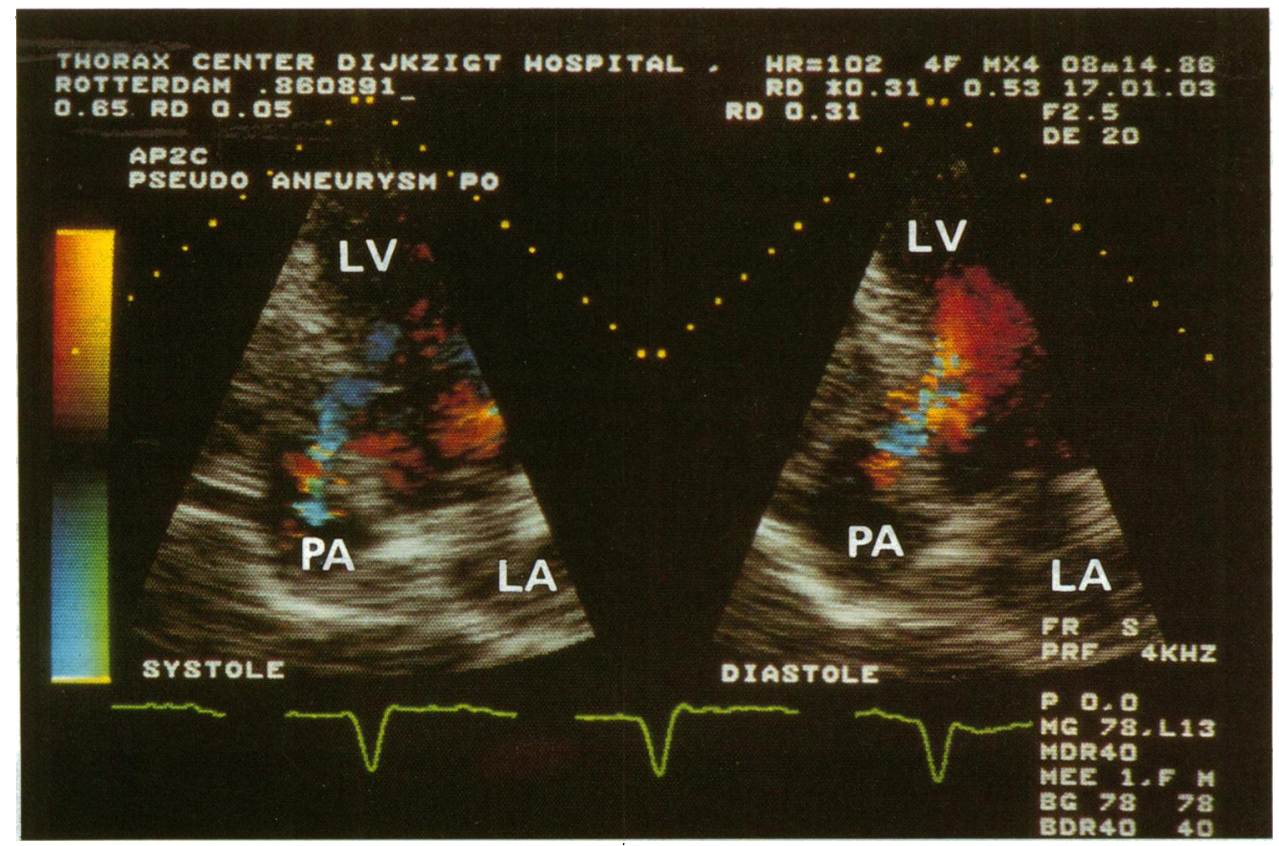

Fig 2 Systolic and diastolic colour flow images (apical long axis view) from a patient with a left ventricular pseudoaneurysm after myocardial infarction (see text). LV, left ventricle; LA, left atrium; $P A$, pseudoaneurysm.

atrial contraction and the second peak with ventricular systole. Flow out of the pseudoaneurysm started in late systole. The maximum velocity peak in this direction was in early to mid diastole. There was a consistent and often pronounced variation in maximal velocity throughout the respiratory cycle, with inspiration increasing the maximal jet velocity by up to $1.5 \mathrm{~m} / \mathrm{s}$. The waveform had a similar configuration to that of the mitral inflow velocities but differed fundamentally in its timing. This flow pattern was characteristic of all 11 cases of pseudoaneurysm studied; it varied only in the peak velocity of the waveforms (that is, the degree of restriction of the myocardial defect) and their degree of respiratory variation in peak velocity.

\section{DOPPLER COLOUR FLOW IMAGING}

In all 11 cases of left ventricular pseudoaneurysm colour flow imaging demonstrated one or more systolic turbulent jets exiting from the left ventricular cavity through the left ventricular free wall or apex into an echo free cavity bounded by pericardium. Diastolic turbulence was consistently noted within the left ventricular cavity at the site of the defect. Figure 2 shows a colour flow image of a narrow, turbulent, mosaic jet entering and leaving a posterolateral pseudoaneurysm through a break in the lateral wall of the left ventricle. In systole a mosaic pattern was identified within the pseudoaneurysm-confirming turbulent flow entering the pseudoaneurysm. In diastole the mosaic pattern occurred within the left ventricle-confirming turbulent flow exiting from the pseudoaneurysm. The extension of the jet and the degree of turbulence varied with respiration. In one patient two jets were found. In early systole they were directed into the pseudoaneurysm and in early diastole they were directed from the pseudoaneurysm into the left ventricular cavity. The diagnosis of all 11 cases was confirmed by left ventricular angiography or operation or both. During the two year study period there were no false positive diagnoses of left ventricular pseudoaneurysm in more than 9000 routine and emergency cases studied in the two echocardiographic laboratories.

\section{Discussion}

A pseudoaneurysm of the left ventricle is defined as a myocardial rupture contained by pericardium and thrombus with no remnants of myocardial tissue. ${ }^{12}$ It is a rare complication of myocardial infarction, but has also been reported after cardiac surgery, chest trauma, and endocarditis. ${ }^{3}$ A pseudoaneurysm after 
rupture of a true left ventricular aneurysm is rare, although it has been reported as a coincidental finding. ${ }^{11}$ Clinically and radiographically it is difficult to distinguish a pseudoaneurysm from a true aneurysm of the left ventricle-until recently this required left ventricular angiography. The suggested tendency for pseudoaneurysms to rupture ${ }^{4}$ means that non-invasive recognition of these two types of aneurysm is important because their management may differ, especially in symptom free patients. Cross sectional echocardiography is a useful noninvasive technique for the diagnosis of left ventricular pseudoaneurysms, as is radionuclide imaging, ${ }^{12}$ which is not so widely available.

The main echocardiographic features of a left ventricular pseudoaneurysm include: (a) a saccular echo free space outside the perimeter of the left ventricle, which usually contains thrombus; (b) a sharp break in the myocardial echo; and $(c)$ an entry orifice with a diameter that is small compared with the diameter of the pseudoaneurysm cavity. This contrasts with true left ventricular aneurysms where the diameters of the orifice and cavity are approximately equal. ${ }^{67}$ However, cross sectional echocardiography may fail to identify small myocardial rupture sites. The differential diagnosis is then a pseudoaneurysm, a localised pericardial effusion or haematoma, and a pericardial cyst or diverticulum. In theory the improved image resolution obtained by transoesophageal echocardiography might improve the identification of small ruptures of the myocardium not visualised by conventional echocardiography. Experience with transoesophageal studies of left ventricular pseudoaneurysms is limited. In two cases in our series transoesophageal echocardiography was performed with the patient anaesthetised before cardiopulmonary bypass. Both were cases of posterolateral pseudoaneurysm and the rupture sites could not be directly visualised in either case. This may reflect the inherent difficulty in imaging the posterolateral and apical aspects of the left ventricle by the transoesophageal approach. Further experience is required before a more definitive statement can be made about any benefits which might be gained by using this new technique in the diagnosis of left ventricular pseudoaneurysm.

The addition of pulsed Doppler has proved a helpful adjunct to cross sectional echocardiography because it increases diagnostic specificity and sensitivity. ${ }^{910}$ Pulsed Doppler confirms abnormal flow between the left ventricle and the echo free space at the site of myocardial disruption, thus distinguishing it from echo "drop-out" and can confirm the diagnosis even when the site of myocardial rupture is not visualised. However, in our study the combination of cross sectional and Doppler echocardiography failed to establish the diagnosis in $27 \%$ of cases. In contrast, colour flow imaging showed the communication between the left ventricle and the pseudoaneurysm in all 11 cases. The resultant turbulent jet was used to delimit the area in which continuous wave and pulsed Doppler studies could confirm the characteristic waveform. In addition, colour flow imaging provided information on the flow characteristics of blood within the pseudoaneurysm. Flow was directed along one wall of the pseudoaneurysm towards its most distal point where it turned and then returned along the other wall. The area of disturbed flow was usually restricted to the area around the entry/exit point of the pseudoaneurysm; flow was laminar around the edges.

Echocardiography often shows thrombus within the pseudoaneurysm cavity. ${ }^{6-8} \mathrm{We}$ found thrombus in half the patients we studied; it seemed to be more common in patients with chronic pseudoaneurysms than in those with acute ones.

In nine cases we were able to compare the results of non-invasive tests with those obtained at cardiac catheterisation. In seven cases left ventricular angiography produced good opacification and delineation of the pseudoaneurysm cavity with identification of the "neck" of the pseudoaneurysm. In the other two cases opacification and identification of the "neck" of the pseudoaneurysms were not possible because of dilution of contrast within their large cavities. In these cases the diagnosis was confirmed only by the appearance of the faintest trace of contrast outside the left ventricular contour. Others have also reported that some left ventricular angiograms are unsatisfactory for the diagnosis of left ventricular pseudoaneurysm. ${ }^{6}$

We conclude that colour flow imaging is a sensitive diagnostic technique that should be in addition to cross sectional imaging and conventional Doppler echocardiography for the diagnosis of left ventricular pseudoaneurysm. Cardiac catheterisation can then be reserved for those patients who require precise coronary artery definition before surgical repair.

\section{References}

1 Hurst CO, Fine G, Keyes J. Pseudoaneurysm of the heart. Circulation 1963;28:427-36.

2 Roberts WC, Morrow AG. Pseudoaneurysm of the left ventricle. An unusual sequel of myocardial infarction and rupture of the heart. $A m J$ Med 1967;43: 639-44.

3 Davidson KH, Parisi AF, Harrington JJ, Barsamian EM, Fishbein MC. Pseudoaneurysm of the left ventricle: an unusual echocardiographic presentation. Review of the literature. Ann Intern Med 1977; 86:430-3. 
4 Van Tassel RA, Edwards JE. Rupture of heart complicating myocardial infarction. Chest 1972;61: 104-16.

5 Vlodaver Z, Coe JI, Edwards JE. True and false aneurysms: propensity of the latter to rupture. Circulation 1975;51:567-72.

6 Gatewood RP, Nanda NC. Differentiation of left ventricular pseudoaneurysm from true aneurysm with two-dimensional echocardiography. Am J Cardiol 1980;46:869-77.

7 Catherwood E, Mintz GS, Kotler MN, Parry WR, Segal BL. Two-dimensional echocardiographic recognition of left ventricular pseudoaneurysm. Circulation 1980;62:294-303.

8 Saner HE, Asinger RW, Daniel JA, Olson J. Twodimensional echocardiographic identification of left ventricular pseudoaneurysm. Am Heart J 1986; 112:977-85.

9 Grube E, Redel D, Jansen R. Non-invasive diagnosis of a false left ventricular aneurysm by echocardiography and pulsed Doppler echocardiography. Br Heart J 1980;43:232-6.

10 Loperfido F, Pennestri F, Mazzari M, et al. Diagnosis of left ventricular pseudoaneurysm by pulsed Doppler echocardiography. Am Heart J 1985;110:1291-3.

11 Coupe M, Dancy M, Pepper J. Coincidence of true and false left ventricular aneurysms after myocardial infarction. Br Heart $J$ 1986;56:567-8.

12 Botvinick EH, Shames D, Hutchinson JC, Roe BB, Fitzpatrick M. Non-invasive diagnosis of a false left ventricular aneurysm with radioisotope gated cardiac blood pool imaging. Am J Cardiol 1976;37:1089-93. 